\title{
Knowledge and judgments about events that occurred prior to birth: The measurement of the persistence of information
}

\author{
DAVID C. RUBIN \\ Duke University, Durham, North Carolina
}

\begin{abstract}
Data from five laboratories using five different techniques were reanalyzed to measure subjects' knowledge of events that occurred over the past 70 years. Subjects were about 20 years of age, so the measures included events that extended up to 50 years before birth. The functions relating knowledge about the events to age do not decrease precipitously at birth but gradually drop to above-chance levels. Techniques usually used to study retention within the individual can be used to study the persistence of ideas and fashions within an age cohort in a culture.
\end{abstract}

Psychologists have studied retention extensively using a variety of techniques, including questions about events that occurred outside the laboratory (Rubin \& Wenzel, 1996). The purpose of this paper is to use such techniques to measure the persistence of cultural or historical information by examining information for events that occurred prior to the birth of the person being tested.

In questionnaire studies, younger adults often are asked questions about events that occurred before their birth to demonstrate that younger adults do not do as well as older adults who experienced and therefore have episodic memory for the events. Consider one carefully conducted study by Botwinick and Storandt (1980) in which groups of 20 subjects with the nominal ages of $25,35,45,55,65$, and 75 were asked questions about entertainment and current events, which were distributed uniformly across the oldest subjects' lives. The same questions were asked for recall and then as part of a five-choice recognition test. Botwinick and Storandt's youngest three groups were asked questions about events that occurred over a span of at least 20 years before they were born. By combining these three groups' data, fairly smooth curves were obtained for both the recall and the recognition questions. As is shown in Figure 1, the younger adults do much better for periods from which they were alive, but have some information about events that occurred before their birth. The recall scores remain well above $0 \%$, and the recognition scores well above a $20 \%$ chance level, assuming all distracters were equally plausible to the target item. Botwinick and Storandt's younger participants' knowledge of the events that occurred before their birth is a measure of the history maintained by their culture. Unlike the events that occurred after birth, which could have both direct and indirect experience as sources of learning (Larsen, 1988), the

I thank Erik Bergman and the editor for their help. Reprints are available from D. C. Rubin, Department of Experimental Psychology, Duke University, Durham, NC 27708-0086 (e-mail: rubin@psych.duke.edu). events from before birth could have only indirect sources of learning.

Before examining the issue of cultural persistence in more detail, several additional studies and experimental methods are presented. If enough subjects and questions are included, individual domains can be examined, as is shown in Figure 2. Rubin, Rahhal, and Poon (1998) asked 60 undergraduates to answer questions about three domains of knowledge: (1) the Academy Awards, as measured by a question on who and also a question on what movie won the Academy Award in each year; (2) the World Series, as measured by asking which two teams played in the World Series; and (3) current events, as measured by a question on what was the most important news story of the year according to the Associated Press and also by a question on who lost the presidential election for years in which there was one. A five-choice recognition test was used in which the four foils were designed to be graded in difficulty by using an algorithm. For example, the four baseball foils included the teams in second place in both leagues, the teams in last place, and the teams with two degrees of success in between. Half the subjects were tested in 1984 and half in 1994, in order to try to partly unconfound the age of the subject and the particular question asked, something Botwinick and Storandt (1980) did by using subjects of different ages. Figure 2 shows the results against a $20 \%$ chance baseline. Note that there are clear differences in the domains. The Academy Award questions start well above the World Series ones but drop to chance, whereas the World Series questions level off at about $8 \%$ above chance.

Judgments of importance and preference show a similar effect. Schuman and Rieger (1992, p. 325), as part of a longer phone survey, asked about 100 people in each 5year age grouping covering the adult lifespan the following question. "There have been a lot of national and world events and changes over the past half century - from 1930 right up until today. Would you mention one or two national or world events or changes that seem to you to have 


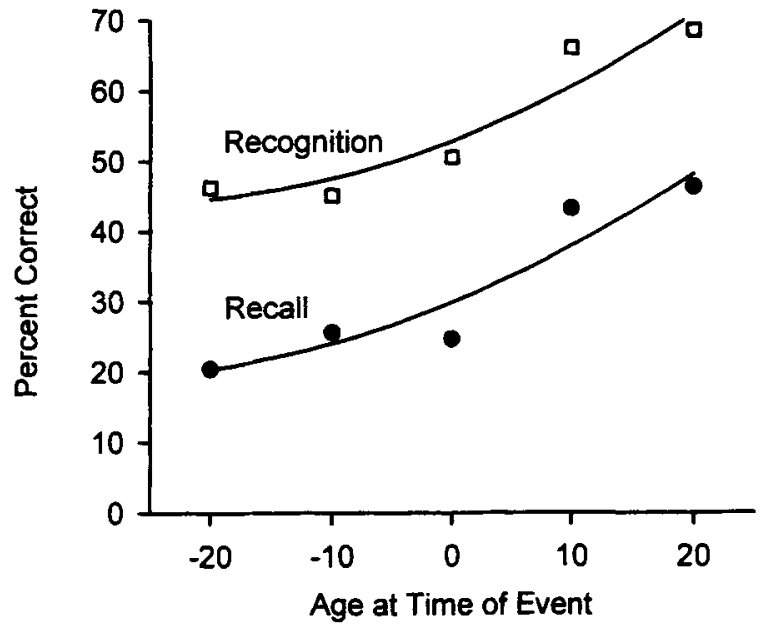

Figure 1. Recall and recognition scores from Botwinick and Storandt (1980). Chance for the recognition is at $20 \%$. The curves are second-order regression fits.

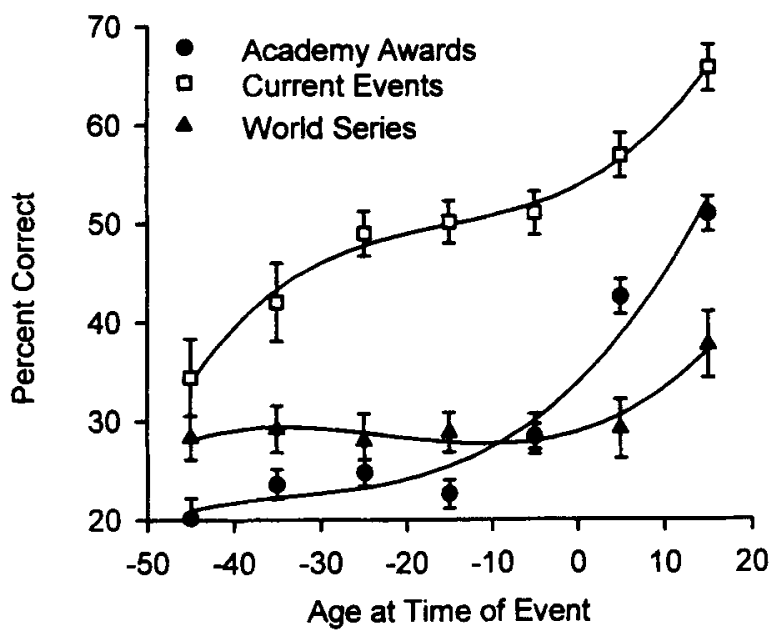

Figure 2. Recognition scores for three domains of knowledge, with standard errors, from Rubin, Rahhal, and Poon (1998). Chance is at $20 \%$. The curves are third-order regression fits.

been especially important." Figure 3 shows the percentage of respondents who answered with either the Depression or World War II, plotted as a function of their age at the middle of these events. Later events, such as the Vietnam War and JFK's assassination did not have respondents who were alive 20 years before these events at the time of the testing. In this analysis, instead of each subject responding to many questions that occurred at different times, each subject provided data at two values on the $x$-axis, in that each subject either responded or did not respond with one of the two events. The location of these data on the $x$-axis depends on the subject's age at the time of the Depression and World War II. In spite of this difference and the change from the request for accurate information to a judgment of importance, the same basic effect occurs. There is a drop that continues for periods before birth, rather than a sharp and complete drop at birth. Whereas the previous examples were about memory for factual, historical information, this study is about what facts subjects thought should be considered as history.

Holbrook and Schindler (1989) show a similar finding for popular music. Here, subjects ranging in age from 16 to 86 were asked to rate how much they liked 28 popular songs, 1 from each evenly numbered year between 1932 and 1986. For each individual, these ratings were converted to $z$ scores and averaged. Because both the age of the subjects and the year each song was popular were uniformly distributed, individuals and individual songs enter into data points all along the $x$-axis. Thus, the quality of individual songs cannot be causing the effect. Figure 4 groups Holbrook and Schindler's year-by-year data into 10 -year intervals. The standard errors are calculated over the 10 years of each period, not over subjects. Again there is a gradual prebirth decline. The study of popular music makes another point. What is being termed here as culture changes over time. The data obtained for one cohort cannot be blindly applied to another. For some domains, there is more change than others, but even in domains as seemingly stable as history, there are changes (Frisch, 1989), which brings us to our final figure.

Cultural persistence can even be measured, using the free recall of long lists of items. Roediger and Crowder (1976) asked undergraduates to recall all of the presidents of the United States that they could. Figure 5 shows the results for their position-recall-criterion measure, which minimizes the increased recall of presidents who had the same last name as other presidents. Assume that, if Roediger and Crowder tested undergraduates during the term of any of the presidents represented by a dot in

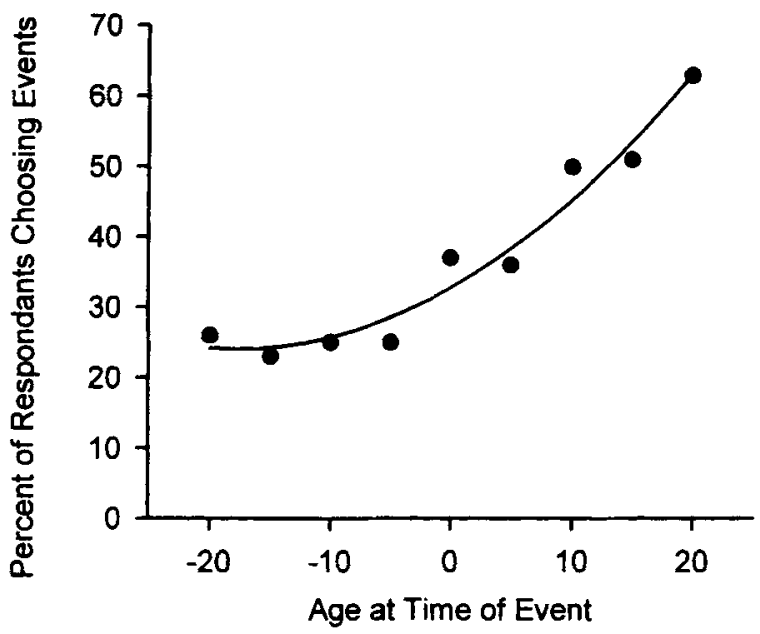

Figure 3. The percentage of respondents who answered with either the Depression or World War II as the most important event of the last 50 years, as a function of their age at the time of the events, from Schuman and Rieger (1992). The curve is a secondorder regression fit. 


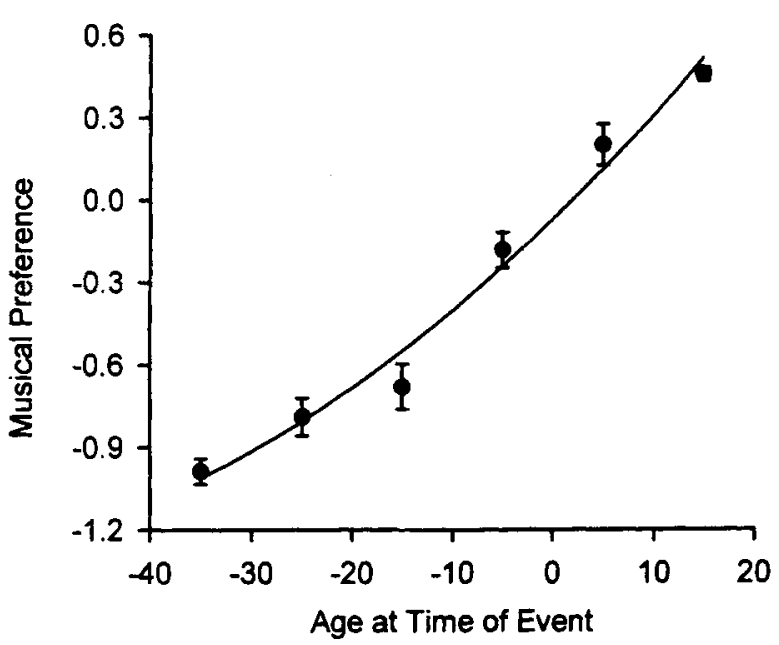

Figure 4. Preference for popular songs, from Holbrook and Schindler (1989). The standard errors were calculated from the 10 data points reported for each period. The curve is a secondorder regression fit.

Figure 5 , the recall of that president would be near $100 \%$. The curve then shows a smooth drop in cultural persistence for 70 years, 50 of them prior to the birth of the undergraduates. However, if the smooth monotonic decline of Figure 5 were continued back for a century before the undergraduates' birth, there would be an increase at the time of President Lincoln, and, if it were continued back for two centuries before birth, there would be an increase for the founding fathers. For these cases, the cultural persistence coincides with formal history. Roediger and Crowder interpreted their result in terms of primacy and recency. Recency may be another term for retention, and primacy can be seen here as mediated by the importance culture and formal histories place on beginnings.

The five figures can now be examined for both abovechance performance and the shape of the function for events before and after the birth of the subjects. Figure 4 cannot be included in the chance performance comparison, because it shows preference ratings and not the knowledge that an event occurred. For all other figures, there is a clear above-chance performance at some of the points before birth, if one assumes that chance recall performance is near zero and that standard errors in studies that do not report them are reasonable for the number of subjects they have. That is, for the domains tested, people know about events not experienced directly in their lifetime.

A description of the shape of the function is more difficult. In general, assuming there is both personally experienced episodic and culturally transmitted semantic knowledge, one might expect to see a steeper decline for episodic memory for experienced events superimposed on a slower decline for shared, retold, and therefore rehearsed events the culture maintains, whether or not they were experienced. Thus, we might see shallower declines before birth where there is no directly experienced episodic memory. The use of regression fits in the figures emphasizes continuity. For Figures 1 and 3 and the lower two curves in Figure 2, but not the other figures, one could also have easily drawn a horizontal line at a level above chance through all events that occurred before the subjects were born and a monotonically decreasing function for events that occurred within the subjects' lifetimes. Decisions between these and among other descriptions of the data are difficult and would be even with laboratory data, as logarithmic, power, and exponential functions would all appear to level off at some point. Such decisions could be made with more certainty if there were small standard errors for all data and longer periods of measurement before birth to see if the curve eventually declined to chance levels. It is clear from the data already assembled, however, that different domains and possibly different methods reveal different time courses for the preservation of cultural information. Not all fame comes and goes in a minute. Some has a considerably longer halflife. Although knowledge of Academy Awards from before the subjects' lifetimes appears to fade quickly, a host of political and current event information does not. A small residue of fame appears permanent in baseball.

Methods usually used to study memory within the individual can also be used to study the persistence of ideas and preferences outside the lifetime of the individual. In this way, the persistence of ideas within a culture can be quantified. The study of memory and history have much in common. "The challenge of history is to recover the past and introduce it to the present. It is the same challenge that confronts memory" (Thelen, 1989, p. 1117). The techniques psychologists have developed for one can be used to study the other. Although the question of cultural transmission has been separated from the question of personal memory and is no longer one of current interest to mainstream memory research, it was one that is part of our history (Bartlett, 1932; Rubin, 1995).

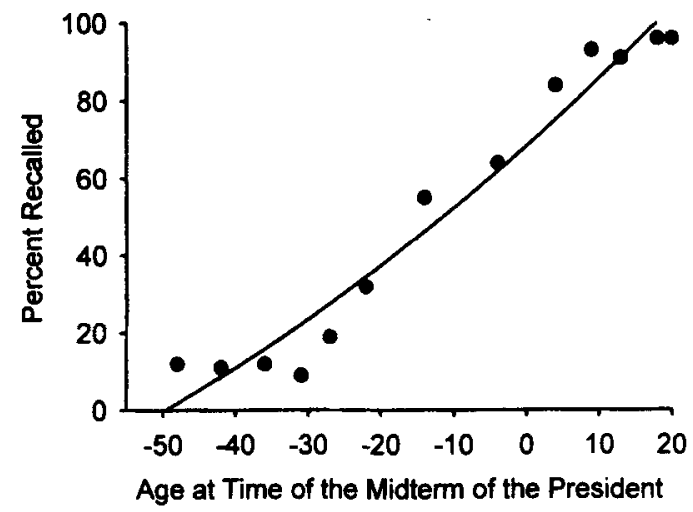

Figure 5. The percentage of subjects who recalled each president since 1900, from Roediger and Crowder (1976). The subjects' years of birth were assumed to be 1953 . The curve is a secondorder regression fit. 
Isolating nonepisodic cultural or semantic memory in questions about events also has direct application to current psychological research. For instance, to the extent that the curves shown in Figures $1-5$ used continuous functions to describe knowledge of events that occurred both before and after birth and to the extent that the level of performance on events that occurred before birth exceeds chance levels, the assumption that event questionnaires study memory for experienced, time-specific episodes is called into question. At a more theoretical level, Neisser (1982) has argued that flashbulb and other memories are a way of tying an individual's history to history. To test and use this and similar ideas, a knowledge of what that individual considers as history is needed. More generally, all studies of autobiographical memory need to differentiate which aspects of memory for experiences are shared and which are the individual's. When people remember personally experienced events, they have many sources of memory and possibly use different memory systems (Brewer, 1986; Larsen, 1988). Examining memories for events that could not have been personally experienced provides one tool that can be used to tease apart these sources and systems.

\section{REFERENCES}

BARTLETT, F. C. (1932). Remembering: A study in experimental and social psychology. London: Cambridge University Press.

Botwinick, J., \& STORANDT, M. (1980). Recall and recognition of old information in relation to age and sex. Journal of Gerontology, 35, 70-76
BREWER, W. F. (1986). What is autobiographical memory? In D. C. Rubin (Ed.), Autobiographical memory (pp. 25-49). Cambridge: Cambridge University Press.

FrisCH, M. (1989). American history and the structure of collective memory: A modern exercise in empirical iconography. Journal of American History, 75, 1130-1155.

HolBROOK, M. B., \& SCHINDLER, R. M. (1989). Some exploratory findings on the development of musical tastes. Journal of Consumer Research, 16, 119-124.

LARSEN, S. F. (1988). Remembering without experiencing: Memory for reported events. In U. Neisser \& E. Winograd (Eds.), Remembering reconsidered: Ecological and traditional approaches to the study of memory (pp. 326-355). Cambridge: Cambridge University Press.

NeISSER, U. (1982). Snapshots or benchmarks? In U. Neisser (Ed.), Memory observed (pp. 43-48). San Francisco: Freeman.

RoEDIGER, H. L., III, \& CROWDER, R. G. (1976). A serial position effect in recall of United States presidents. Bulletin of the Psychonomic Society, 8, 275-278.

RuBiN, D. C. (1995). Memory in oral traditions: The cognitive psychology of epic, ballads, and counting-out rhymes. New York: Oxford University Press

Rubin, D. C., Rahhal, T. A., \& Poon, L. W. (1998). Things learned in early adulthood are remembered best. Memory \& Cognition, 26, 3-19.

RuBIN, D. C., \& WENZEL, A. E. (1996). One hundred years of forgetting: A quantitative description of retention. Psychological Review, 103, 734-760.

Schuman, H., \& Rieger, C. (1992). Collective memory and collective memories. In M. A. Conway, D. C. Rubin, H. Spinnler, \& W. A. Wagenaar (Eds.), Theoretical perspectives on autobiographical memory (pp. 323-336). Dordrecht: Kluwer.

THELEN, D. (1989). Memory and American history. Journal of American History, 75, 1117-1129.

(Manuscript received August 7, 1997; revision accepted for publication January 12,1998 .) 